\title{
High-dose intravenous selenium does not improve clinical outcomes in the critically ill: a systematic review and meta-analysis
}

\author{
William Manzanares ${ }^{* *}$, Margot Lemieux ${ }^{2}$, Gunnar Elke ${ }^{3}$, Pascal L. Langlois ${ }^{4}$, Frank Bloos ${ }^{5}$ and Daren K. Heyland ${ }^{2,6}$
}

\begin{abstract}
Background: Selenium (Se) is an essential trace element with antioxidant, anti-inflammatory, and immunomodulatory effects. So far, several randomized clinical trials (RCTs) have demonstrated that parenteral Se may improve clinical outcomes in intensive care unit (ICU) patients. Since publication of our previous systematic review and meta-analysis on antioxidants in the ICU, reports of several trials have been published, including the largest RCT on Se therapy. The purpose of the present systematic review was to update our previous data on intravenous (IV) Se in the critically ill.

Methods: We searched MEDLINE, Embase, and the Cochrane Central Register of Controlled Trials. We included RCTs with parallel groups comparing parenteral Se as single or combined therapy with placebo. Potential trials were evaluated according to specific eligibility criteria, and two reviewers abstracted data from original trials in duplicate independently. Overall mortality was the primary outcome; secondary outcomes were infections, ICU length of stay (LOS), hospital LOS, ventilator days, and new renal dysfunction.

Results: A total of 21 RCTs met our inclusion criteria. When the data from these trials were aggregated, IV Se had no effect on mortality (risk ratio [RR] $0.98,95 \% \mathrm{Cl} 0.90-1.08, P=0.72$, heterogeneity $P^{2}=0 \%$ ). In addition, when the results of ten trials in which researchers reported on infections were statistically aggregated, there was no significant treatment effect of parenteral Se (RR $0.95,95 \% \mathrm{Cl} 0.88-1.02, P=0.15, I^{2}=0 \%$ ). There was no positive or negative effect of Se therapy on ICU and hospital LOS, renal function, or ventilator days.
\end{abstract}

Conclusions: In critically ill patients, IV Se as monotherapy does not improve clinical outcomes.

Keywords: Parenteral selenium, Intravenous selenium, Antioxidant micronutrient, Critically ill

\section{Background}

Selenium (Se) is an essential trace element with antiinflammatory and immunomodulatory properties, and it is currently considered the cornerstone of the antioxidant defense system $[1,2]$. Over the past 30 years, a large number of basic and clinical studies have revealed the crucial role of Se in the maintenance of immune, metabolic, endocrine, and cellular homeostasis, which is attributed to its presence in selenoproteins, as the 21st amino acid selenocysteine [3]. These selenoenzymes are involved in redox signaling, antioxidant defense, thyroid hormone metabolism, and immune responses [4]. Thus

\footnotetext{
* Correspondence: wmanzanares@adinet.com.uy

'Department of Critical Care, Intensive Care Unit, Hospital de Clínicas (University Hospital), Faculty of Medicine, Universidad de la República (UdelaR), Avenida Italia, 14th Floor, Montevideo 11.600, Uruguay Full list of author information is available at the end of the article
}

far, critical illness with systemic inflammation and multiple organ dysfunction syndrome was deemed to be associated with an early reduction in plasma/serum Se and glutathione peroxidase (GPx) activity, where both parameters correlate inversely with the severity of illness and clinical outcome $[5,6]$.

Over the last two decades, researchers in several randomized clinical trials (RCTs) have evaluated the role of parenteral inorganic selenocompounds such as sodium selenite or selenious acid, either as a single-agent strategy or in combination with other antioxidant micronutrients (antioxidant cocktails) and using different dose regimens in critically ill patients with systemic inflammation. These studies have shown beneficial results in terms of reduction of infections, mortality, and other relevant clinical outcomes in the critically ill. 
In 2005, the authors of the first comprehensive systematic review and meta-analysis on antioxidant nutrients in the critically ill [7] demonstrated that Se supplementation could be associated with a reduction in mortality, while nonselenium antioxidants had no effect on mortality. More recently, authors of other systematic reviews and meta-analyses [8-14] on Se therapy in intensive care unit (ICU) patients found that pharmaconutrition with parenteral Se monotherapy may be able to significantly reduce mortality in patients with sepsis, particularly when an intravenous (IV) bolus was provided and daily doses higher than $500 \mu \mathrm{g}$ were administered. In addition, Se substitution was more effective in those patients with higher risk of death [13].

Nonetheless, since 2013, several studies of the effects of parenteral Se supplementation as single or combined therapy have been published [7, 14-17]. The REducing Deaths due to Oxidative Stress (REDOXS) trial [16] investigators were unable to find a therapeutic benefit of a combined Se supplementation regimen $(300 \mu \mathrm{g}$ enteral plus $500 \mu \mathrm{g}$ parenteral). The most recent and largest RCT on Se monotherapy in severe sepsis and septic shock, the Sodium Selenite and Procalcitonin Guided Antimicrobial Therapy in Severe Sepsis (SISPCT) study [17], further demonstrated that high-dose IV sodium selenite was not associated with improved survival. Therefore, with the aim of elucidating the overall efficacy of parenteral Se as single or combined therapy (antioxidant cocktails) in adult critically ill patients, we performed an update of our previous systematic review and meta-analysis of the literature.

\section{Methods}

\section{Study identification}

We conducted a systematic review of the literature published between 1980 and 2015 using the computerized databases of MEDLINE, Embase, the Cochrane Controlled Trials Register, and the Cochrane Database of Systematic Reviews. Text words or $\mathrm{MeSH}$ headings containing "randomized," "blind," "clinical trial," "parenteral," "intravenous," "selenium," "sodium selenite," "selenious acid," "antioxidant cocktails," "critical illness," and "critically ill" were used without any language restriction. We also reviewed our personal files and comprehensive reviews for additional original studies.

\section{Study selection criteria}

Original studies were included if they met the following criteria:

1. Randomized controlled trial study design with a parallel group

2. A population of critically ill adult patients ( $>18$ years old), defined as patients admitted to an ICU (If the study population was unclear, we considered a mortality rate higher than $5 \%$ in the control group to be consistent with critical illness.)

3. The administration of parenteral Se in the intervention arm (either with or without an initial bolus) as a single-agent strategy or in combination with other antioxidant micronutrients compared with a control group with a placebo

4. The evaluation of clinically relevant outcomes such as mortality, infectious complications, ICU or hospital length of stay (LOS), length of mechanical ventilation (MV), and new renal dysfunction including the requirement of renal replacement therapy

Clinical studies that reported only biochemical, metabolic, or immunologic results were excluded.

All original studies were evaluated and abstracted in duplicate independently by two reviewers using a data abstraction form that had been used previously [18]. A discussion was held and consensus was obtained between the reviewers when a disagreement occurred. When additional data were needed, we attempted to contact the authors of the published article. We scored the methodological quality of the original trials, aiming to obtain a score between 0 and 14, with the following high-quality criteria: (1) the extent to which randomization was concealed, (2) intention-to-treat (ITT)-based analysis, (3) extent of blinding, (4) baseline comparability of groups, (5) extent of follow-up, (6) description of treatment protocols and cointerventions in both arms, and (7) definition of clinical outcomes [18].

We designated studies as level I if all of the following criteria were fulfilled: concealed randomization, blinded outcome adjudication, and an ITT analysis, which are the strongest methodological tools to reduce bias. A study was considered as level II if any one of the abovedescribed characteristics was unfulfilled.

\section{Data analysis}

The primary outcome was overall mortality. Hospital mortality, when available, was used for the statistical analysis. If not reported, we used ICU mortality or 28day mortality. When not specified, mortality was assumed to be hospital mortality. Secondary outcomes included infections, hospital and ICU LOS, MV days, and new renal dysfunction as defined by the authors of the original articles. We used the definition of infections employed by each author. The data from all trials reporting the specific outcome were combined to calculate the pooled risk ratio (RR) for mortality and infections, and pooled weighted mean difference (WMD) for LOS, both with 95 \% CIs. All analyses were conducted using Review Manager (RevMan) 5.3 software, except for the test for asymmetry. Pooled RRs were calculated using 
the Mantel-Haenszel estimator, and WMDs were estimated by the inverse variance approach. The random effects model of DerSimonian and Laird [19] was used to estimate variances for the Mantel-Haenszel and inverse variance estimators. When possible, studies were aggregated on an ITT basis. Heterogeneity in the data was tested by a weighted Mantel-Haenszel chi-square test and quantified by using the $I^{2}$ statistics implemented in RevMan 5.3 software. Differences between subgroups were analyzed using the test of subgroup differences described by Deeks et al. [20], and the results were expressed using the $P$ values. Funnel plots were generated to assess the possibility of publication bias, and the Egger regression test was used to measure funnel plot asymmetry [21]. Asymmetry was calculated using Comprehensive Meta-Analysis 3.0 statistical software (Biostat Inc., Englewood, NJ, USA). $P$ values $<0.05$ and $<0.10$ were considered as statistically significant and indicators of a trend, respectively.

\section{A priori hypothesis testing}

Significant differences in the protocols of the original studies were expected. Thus, several prespecified hypothesisgenerating subgroup analyses were performed to identify potentially more beneficial treatment strategies. First, we compared the results of trials in which investigators administered parenteral Se as monotherapy with studies in which researchers provided parenteral Se in antioxidant cocktails. Based on previous RCTs showing a beneficial effect of an initial loading dose, those RCTs using an initial loading dose as an IV bolus of Se were then compared with trials those that did not. In addition, because researchers in previous trials found that daily doses higher than $500 \mu \mathrm{g}$ were associated with better outcomes, we compared the results between three subgroups having different daily doses: lower than $500 \mu \mathrm{g}$, equal to $500 \mu \mathrm{g}$, and greater than $500 \mu \mathrm{g}$. Moreover, on the basis of a possibly larger treatment effect in patients with higher risk of death, we compared studies including patients with higher mortality vs. those with lower mortality. Mortality was considered to be high or low based on whether it was greater or less than the mean control group mortality of all the trials. Additionally, we postulated that trials with lower quality (level II studies) might demonstrate a greater treatment effect than those trials with higher quality (level I studies). Furthermore, as current evidence showed benefits in terms of reduction in mortality in septic patients, the results of RCTs performed only with patients with sepsis were compared with RCTs performed with heterogeneous patient populations (nonsepsis studies). We also assessed the effect of Se in soils according to the geographical region where the trial was conducted. For this purpose, we compared RCTs performed in deprived regions (Europe, South America, and Asia) versus trials performed in nondeprived regions
(North America). Finally, given the interaction between Se and procalcitonin (PCT) in the SISPCT study [17], we conducted a sensitivity analysis excluding the PCT guidance group of patients.

\section{Results \\ Study identification and selection}

A total of 41 relevant citations were identified in the search of computerized bibliographic databases and a review of reference lists in related articles. Of these, we excluded 20 for the following reasons: 8 trials did not include ICU patients (mostly surgery patients) [22-29]; 1 study did not evaluate clinical outcomes [30]; 1 study compared high-dose with low-dose Se [31]; 3 articles were duplicates [32-34]; 4 articles were systematic reviews; 1 trial was published as an abstract [35], and we were unable to obtain the data from the authors to complete our data abstraction process; 1 study was not an RCT [36]; and in 1 trial Se was not given intravenously [37].

Ultimately, 21 studies [14-17, 38-54] met our inclusion criteria and were included; they comprised a total of 4044 patients (Tables 1 and 2). The reviewers reached $100 \%$ agreement for the inclusion of the trials. The mean methodological score of all trials was 9 of a maximum possible score of 14 (range 4-13). Randomization was concealed in 9 (43\%) of 21 trials; ITT analysis was performed in 14 (67\%) of 21 trials; and double-blinding was done in 7 (33\%) of 21 of the studies. There were 6 level I studies and 15 level II studies. The details of the methodological quality of the individual trials are shown in Table 1.

\section{Primary outcome: mortality}

When the results of the 21 trials in which researchers reported mortality were aggregated, no statistically significant difference was found between Se supplementation and placebo (RR 0.98, $95 \%$ CI $0.90-1.08, P=0.72$, heterogeneity $I^{2}=0 \%$ ) (Fig. 1). In the sensitivity analysis, after excluding the PCT guidance group of the Bloos et al. study, we found that the new RR was 0.95 (95\% CI $0.79-1.15, P=0.63$, $I^{2}=35 \%$ ) (Additional file 1: Figure S1).

\section{Secondary outcomes \\ Overall effect on new infectious complications}

When data from ten studies were included in the metaanalysis, no significant effect of Se supplementation on infections was found (RR 0.95, $95 \%$ CI 0.88-1.02, $P=0.15, I^{2}=0 \%$ ) (Fig. 2).

\section{Overall effect on ICU and hospital length of stay and ventilator days}

When ten RCTs in which researchers reported ICU LOS were statistically aggregated, there were no significant differences between the groups (WMD 0.32, $95 \%$ CI -0.80 
Table 1 Randomized clinical trials evaluating selenium supplementation in critically ill patients

\begin{tabular}{|c|c|c|c|}
\hline Study & Population & Methodology (score) & Intervention \\
\hline Kuklinski 1991 [38] & $\begin{array}{l}\text { Patients with acute pancreatic necrosis } \\
n=17\end{array}$ & $\begin{array}{l}\text { C.Random: not sure } \\
\text { ITT: no } \\
\text { Blinding: no } \\
\text { (4) }\end{array}$ & $\begin{array}{l}\mathrm{PN}+\text { selenium supplementation }(500 \mu \mathrm{g} / \text { day }) \\
\text { vs. PN without selenium supplementation }\end{array}$ \\
\hline Zimmerman 1997 [39] & $\begin{array}{l}\text { Patients with SIRS, sepsis, APACHE II score } \\
>15 \text {, and multiorgan failure score }>6 \\
n=40\end{array}$ & $\begin{array}{l}\text { C.Random: no } \\
\text { ITT: yes } \\
\text { Blinding: no } \\
\text { (6) }\end{array}$ & $\begin{array}{l}\text { IV selenium as sodium selenite } 1000 \mu \mathrm{g} \text { as a } \\
\text { bolus and then } 1000 \mu \mathrm{g} \text { sodium selenite } \\
24 \mathrm{~h} \text { as a continuous infusion over } 28 \text { days } \\
\text { vs. standard }\end{array}$ \\
\hline Berger 1998 [40] & $\begin{array}{l}\text { Burns }>30 \% \text { TBSA } \\
n=20\end{array}$ & $\begin{array}{l}\text { C.Random: yes } \\
\text { ITT: yes } \\
\text { Blinding: double blind } \\
\text { (12) }\end{array}$ & $\begin{array}{l}\text { IV copper }(40.4 \mu \mathrm{mol}) \text {, selenium }(159 \mu \mathrm{g}) \text {, } \\
\text { zinc }(406 \mu \mathrm{mol})+\text { standard trace elements } \\
\text { vs. standard trace elements (copper } \\
20 \mu \text { mol, selenium } 32 \mu \mathrm{g} \text {, zinc } 100 \mu \mathrm{mol}) \\
\text { from days } 0 \text { to } 8 \text {, all received early EN }\end{array}$ \\
\hline Angstwurm 1999 [41] & $\begin{array}{l}\text { Patients with SIRS and sepsis at } 11 \text { ICUs } \\
n=42\end{array}$ & $\begin{array}{l}\text { C.Random: not sure } \\
\text { ITT: yes } \\
\text { Blinding: no } \\
\text { (10) }\end{array}$ & $\begin{array}{l}\text { PN with high-dose selenium }(535 \mu \mathrm{g} \times \\
3 \text { days, } 285 \mu \mathrm{g} \times 3 \text { days, } 155 \mu \mathrm{g} \times 3 \text { days, } \\
\text { and } 35 \mu \mathrm{g} \text { thereafter) vs. low-dose selenium } \\
(35 \mu \mathrm{g} / \text { day for duration of study) }\end{array}$ \\
\hline Porter 1999 [42] & $\begin{array}{l}\text { Surgical ICU penetrating trauma patients } \\
\text { with Injury Severity Score } \geq 25 \\
n=18\end{array}$ & $\begin{array}{l}\text { C.Random: yes } \\
\text { ITT: yes } \\
\text { Blinding: no } \\
\text { (9) }\end{array}$ & $\begin{array}{l}50 \mu \mathrm{g} \text { selenium IV every } 6 \mathrm{~h}+400 \mathrm{IU} \\
\text { vitamin } \mathrm{E}, 100 \mathrm{mg} \text { vitamin } \mathrm{C} \text { every } 8 \mathrm{~h} \text {, and } \\
8 \mathrm{~g} \text { of } \mathrm{N} \text {-acetylcysteine every } 6 \mathrm{~h} \text { via } \\
\text { nasogastric or oral route from days } 0 \text { to } 7 \\
\text { vs. none }\end{array}$ \\
\hline Berger 2001 [43] & $\begin{array}{l}\text { Trauma patients, surgical ICU } \\
n=31\end{array}$ & $\begin{array}{l}\text { C.Random: yes } \\
\text { ITT: no } \\
\text { Blinding: double } \\
\text { (9) }\end{array}$ & $\begin{array}{l}\text { IV selenium supplementation ( } 500 \mu \mathrm{g} / \text { day) } \\
\text { vs. placebo (selenium group randomized } \\
\text { further to two groups: } 500 \mu \mathrm{\mu g} \text { selenium alone } \\
\text { vs. } 500 \mu \mathrm{mg} \text { selenium }+150 \mathrm{mg} \text { a-tocopherol + } \\
13 \mathrm{mg} \text { zinc) given slowly for first } 5 \text { days after } \\
\text { injury (all groups received EN) }\end{array}$ \\
\hline Lindner 2004 [44] & $\begin{array}{l}\text { Patients with acute pancreatitis admitted to } \\
\text { the ICU }\end{array}$ & $\begin{array}{l}\text { ITT: no } \\
\text { Blinding: single }\end{array}$ & $\begin{array}{l}\text { IV sodium selenite dose of } 2000 \mu \mathrm{g} \text { on day } \\
1,1000 \mu \mathrm{g} \text { on days } 2-5 \text {, and } 300 \mu \mathrm{g} \text { from } \\
\text { day } 6 \text { until discharge vs. placebo (isotonic } \\
0.9 \% \mathrm{IV} \mathrm{NaCl} \text { solution) }\end{array}$ \\
\hline
\end{tabular}

(9)

Angstwurm 2007 [45] Multicenter mixed ICUs

$n=249$

Burns $>20 \%$ TBSA

$n=21$

Septic shock patients

$n=60$
C.Random: not sure

ITT: no

Blinding: double

(8)

C.Random: not sure

ITT: yes

Blinding: no

(8)

C.Random: not sure

ITT: no

Blinding: double

(8)
$1000 \mu \mathrm{g}$ selenium IV within $1 \mathrm{~h}$ followed by $1000 \mu \mathrm{g}$ selenium for 14 days vs. $\mathrm{NaCl}$ (0.9\%) (all patients received EN or PN)

IV $100 \mathrm{ml}$ of copper $(59 \mu \mathrm{mol})+$ selenium (375 $\mu \mathrm{g}+$ zinc $(574 \mu \mathrm{mol})$ vs. $\mathrm{NaCl}(0.9 \%)$ from admission for 5-15 days

Both groups were on EN

$4000 \mu \mathrm{g}$ selenium IV on day 1 followed by $1000 \mu \mathrm{g}$ selenium for 9 days vs. $\mathrm{NaCl}(0.9 \%)$ (all patients received EN or $\mathrm{PN}$ ) 
Table 1 Randomized clinical trials evaluating selenium supplementation in critically ill patients (Continued)

\begin{tabular}{|c|c|c|c|}
\hline \multirow[t]{4}{*}{ Mishra 2007 [48] } & Septic ICU patients & C.Random: not sure & \multirow{4}{*}{$\begin{array}{l}474 \mu \mathrm{g} \text { selenium IV } \times 3 \text { days followed by } \\
316 \mu \mathrm{g} \times 3 \text { days, } 158 \mu \mathrm{g} \times 3 \text { days, and } \\
31.6 \mu \mathrm{g} \text { thereafter vs. } 31.6 \mu \mathrm{g} \text { selenium } \\
\text { (all patients received EN or PN) }\end{array}$} \\
\hline & \multirow[t]{3}{*}{$n=40$} & ITT: yes & \\
\hline & & Blinding: double & \\
\hline & & (9) & \\
\hline \multirow[t]{4}{*}{ Berger 2008 [49] } & \multirow{4}{*}{$\begin{array}{l}\text { Mixed ICU } \\
n=200\end{array}$} & C.Random: not sure & \multirow{2}{*}{$\begin{array}{l}\text { IV selenium supplementation loading dose } \\
540 \mu \mathrm{g} / \text { day }+ \text { zinc }(60 \mathrm{mg})+\text { vitamin C } \\
2700 \mathrm{mg}+\text { vitamin B } 305 \mathrm{mg}+\text { vitamin E } \\
\text { enteral } 600 \mathrm{mg}+\text { vitamin E } 12.8 \mathrm{mg} \text { IV for } \\
2 \text { days followed by half the dose of all vs. } \\
\text { standard vitamins }\end{array}$} \\
\hline & & ITT: yes & \\
\hline & & Blinding: no & \multirow[t]{2}{*}{ All groups received EN or PN } \\
\hline & & (10) & \\
\hline \multirow[t]{4}{*}{ El-Attar 2009 [50] } & Patients with COPD & C.Random: yes & $\begin{array}{l}\text { IV selenium as sodium selenite } 100 \mu \mathrm{g} / \mathrm{day} \text {, } \\
\text { zinc } 2 \mathrm{mg} / \text { day, and manganese } 0.4 \mathrm{mg} / \text { day } \\
\text { vs. none }\end{array}$ \\
\hline & \multirow[t]{3}{*}{$n=80$} & ITT: yes & \multirow{3}{*}{$\begin{array}{l}\text { Trace elements were administered during } \\
\text { the period on mechanical ventilation }\end{array}$} \\
\hline & & Blinding: yes & \\
\hline & & (12) & \\
\hline \multirow[t]{4}{*}{ Montoya 2009 [51] } & Medical/surgical septic & C.Random: yes & \multirow{4}{*}{$\begin{array}{l}\text { Day } 1 \text { IV sodium selenite } 1000 \mu \mathrm{g} \text {, day } 2 \\
\text { sodium selenite } 500 \mu \mathrm{g} \text {, and thereafter } \\
200 \mu \mathrm{g} \text { during } 7 \text { additional days vs. selenite } \\
100 \mu \mathrm{g} / \text { day }\end{array}$} \\
\hline & ICU patients & ITT: yes & \\
\hline & \multirow[t]{2}{*}{$n=68$} & Blinding: double & \\
\hline & & (7) & \\
\hline \multirow[t]{4}{*}{ Andrews 2011 [52] } & Mixed ICU, multicenter & C.Random: yes & \multirow{4}{*}{$\begin{array}{l}500 \mu \mathrm{g} \text { selenium supplemented PN (12.5 g } \\
\text { nitrogen, } 2000 \mathrm{kcal}) \text { vs. standard PN (12.5 g } \\
\text { nitrogen, } 2000 \mathrm{kcal} \text { ) initiated after ICU } \\
\text { admission (actual median } 2.6 \text { days) for } \\
7 \text { days (actual duration, mean } 4.1 \text { days). }\end{array}$} \\
\hline & \multirow[t]{3}{*}{$n=502$} & ITT: yes & \\
\hline & & Blinding: double blind & \\
\hline & & (13) & \\
\hline \multirow[t]{4}{*}{ Manzanares 2011 [53] } & Septic or trauma patients & C.Random: not sure & \multirow{4}{*}{$\begin{array}{l}\text { IV selenium supplementation loading dose } \\
2000 \mu \mathrm{g}(2 \mathrm{~h}) \text { on day } 1 \text { followed by } \\
1600 \mu \mathrm{g} / \text { day for } 10 \text { days vs. NaCl as placebo }\end{array}$} \\
\hline & \multirow[t]{3}{*}{$n=35$} & ITT: no (except mortality) & \\
\hline & & Blinding: single blind & \\
\hline & & (9) & \\
\hline \multirow[t]{4}{*}{ Valenta 2011 [54] } & Patients with sepsis or SIRS & C.Random: not sure & \multirow{4}{*}{$\begin{array}{l}\text { IV selenium supplementation loading dose } \\
1000 \mu \mathrm{g} \text { on day } 1 \text { followed by } 500 \mu \mathrm{g} / \text { day } \\
\text { for } 5-14 \text { days }+<75 \mu \mathrm{g} / \text { day of sodium } \\
\text { selenite added to } \mathrm{PN} \text { vs. } \mathrm{NaCl}+<75 \mu \mathrm{g} / \text { day } \\
\text { of sodium selenite added to PN }\end{array}$} \\
\hline & \multirow[t]{3}{*}{$n=150$} & ITT: yes & \\
\hline & & Blinding: no & \\
\hline & & (8) & \\
\hline \multirow[t]{4}{*}{ Heyland 2013 [16] } & Multicenter mixed ICUs & C.Random: yes & \multirow{4}{*}{$\begin{array}{l}500 \mu \mathrm{g} \text { selenium via } \mathrm{PN}+300 \mu \mathrm{g} \text { selenium, } \\
20 \mathrm{mg} \text { zinc, } 10 \mathrm{mg} \text {-carotene, } 500 \mathrm{mg} \\
\text { vitamin } \mathrm{E}, 1500 \mathrm{mg} \text { vitamin } C \text { via EN vs. } \\
\text { placebo via PN and EN }\end{array}$} \\
\hline & \multirow[t]{3}{*}{$n=1218$} & ITT: yes & \\
\hline & & Blinding: double & \\
\hline & & $(12)$ & \\
\hline \multirow[t]{4}{*}{ Woth 2014 [14] } & $\begin{array}{l}\text { Mixed ICU, severe septic patients with } \\
\text { multiorgan failure }\end{array}$ & C.Random: not sure & \multirow{4}{*}{$\begin{array}{l}1000-\mu \mathrm{g} / 30 \text { minutes loading dose of sodium } \\
\text { selenite and } 1000-\mu \mathrm{g} / \text { day treatment for a } \\
\text { maximum of } 14 \text { days vs. control group } \\
\text { (not described) }\end{array}$} \\
\hline & \multirow[t]{3}{*}{$n=40$} & ITT: yes & \\
\hline & & Blinding: no & \\
\hline & & (6) & \\
\hline Bloos 2016 [17] & Multicenter mixed ICU patients with severe & C.Random: yes & IV loading dose of $1000 \mu \mathrm{g}$ sodium selenite \\
\hline & sepsis or septic shock in last $24 \mathrm{~h}$ & ITT: yes & $\begin{array}{l}\text { followed by continuous IV of } 1000 \mu \mathrm{mg} \\
\text { sodium selenite daily until ICU discharge or }\end{array}$ \\
\hline & $n=1089$ & Blinding: double & $\begin{array}{l}\text { for } 21 \text { days, whichever comes first vs. } \\
\text { placebo }(\mathrm{NaCl})\end{array}$ \\
\hline
\end{tabular}


Table 1 Randomized clinical trials evaluating selenium supplementation in critically ill patients (Continued)

\begin{tabular}{|c|c|c|c|}
\hline \multirow[t]{4}{*}{ Chelkeba 2015 [15] } & $\begin{array}{l}\text { Mixed ICU patients with severe sepsis and } \\
\text { septic shock }\end{array}$ & C.Random: yes & \multirow{4}{*}{$\begin{array}{l}\text { IV loading dose of } 2000 \mu \mathrm{g} \text { sodium selenite } \\
\text { followed by continuous IV of } 1500 \mu \mathrm{g} \\
\text { sodium selenite daily until day } 14 \mathrm{vs} \text {. } \\
\text { standard therapy without selenium }\end{array}$} \\
\hline & \multirow[t]{3}{*}{$n=54$} & ITT: no & \\
\hline & & Blinding: single & \\
\hline & & (11) & \\
\hline
\end{tabular}

Abbreviations: APACHE Acute Physiology and Chronic Health Evaluation, COPD Chronic obstructive pulmonary disease, C.Random Concealed randomization, $D 5 W$ Dextrose $5 \%$ in water, EN Enteral nutrition, ICU Intensive care unit, ITT Intention to treat, IV Intravenous, PN Parenteral nutrition, SIRS Systemic inflammatory response syndrome, TBSA Total body surface area

to $\left.1.43, P=0.58, I^{2}=3 \%\right)$. When the eight studies in which researchers reported hospital LOS were aggregated, there were no significant differences between the groups (WMD $-0.83,95 \% \mathrm{CI}-3.71$ to $2.04, P=0.57, I^{2}=0 \%$ ). Also, when the eight studies in which researchers reported ventilator days were aggregated, Se supplementation was not associated with a reduction in MV days, although significant heterogeneity was present (WMD -1.75, $95 \%$ CI -4.30 to $0.81, P=0.18, I^{2}=74 \%, P$ value for test of heterogeneity $=0.0004)$.

\section{Overall effect on new renal dysfunction}

After aggregating the data from eight RCTs in which researchers reported new renal dysfunction, Se therapy was not associated with a significant reduction in the incidence of renal dysfunction (RR 0.79, $95 \%$ CI 0.57-1.08, $P=0.14$, $\left.I^{2}=0 \%\right)$.

\section{Subgroup analyses \\ PN selenium monotherapy vs. combined therapy}

The RR associated with parenteral Se monotherapy for mortality was 0.91 (95 \% CI 0.79-1.04, $P=0.16 ; I^{2}=12 \%$ ) (Fig. 1), compared with 1.08 for studies in which researchers used Se as part of combination therapy (95\% CI $0.93-1.25, P=0.33)$. The test of subgroup differences was not significant $(P=0.10)$. There was no effect on infections with either Se monotherapy or combined therapy (test for subgroup differences, $P=0.59$ ) (Fig. 2).

\section{PN selenium loading dose vs. no loading dose}

There was no treatment effect difference in mortality between studies in which researchers used a parenteral loading dose of Se as an IV bolus (RR 0.90, $95 \% \mathrm{CI}$ $0.75-1.08, P=0.30, I^{2}=18 \%$ ) and those not using a loading dose (RR 1.01, $95 \%$ CI $0.90-1.14, P=0.83$ ) (Fig. 3). The test for subgroup differences was not statistically significant $(P=0.30)$. There was also no effect on infectious complications in studies in which researchers used a parenteral loading dose (RR 0.99, 95 \% CI 0.901.09, $P=0.84, I^{2}=0 \%$ ), whereas there was a significant reduction on infections in studies without a parenteral Se loading dose (RR 0.87, $95 \%$ CI $0.77-0.99, P=0.03$, $I^{2}=0 \%$ ) (Fig. 4); the test for subgroup differences was not significant $(P=0.11)$.

\section{PN selenium high dose vs. low dose}

There were no significant treatment effect differences in mortality (test for subgroup differences $P=0.89$ ) and infections (test for subgroup differences $P=0.52$ ) when we compared studies with high-dose vs. low-dose parenteral Se supplementation (data not shown).

\section{Higher vs. lower mortality}

The mean hospital mortality rate (or ICU mortality when hospital mortality was not reported) in the control group of all the trials was $33 \%$. After aggregating 11 studies with a higher mortality rate, we found that IV Se did not have an effect on mortality (RR 0.94, $95 \% \mathrm{CI}$ 0.84-1.05, $\left.P=0.26, I^{2}=17 \%\right)$. In addition, IV Se did not have an effect on mortality in eight studies with a lower mortality (RR 1.08, $95 \%$ CI 0.90-1.08, $P=0.24, I^{2}=0 \%$ ). The test for subgroup differences was not significant $(P=0.17)$ (see Additional file 2: Figure S2) In addition, there were no significant treatment effect differences in infections (test for subgroup differences $P=0.35$ ) when we compared studies with high vs. low mortality rates in the control group (see Additional file 2: Figure S3).

\section{Study quality on outcomes}

When we evaluated study quality on outcomes, a statistically significant effect of IV Se on the reduction of mortality was found in the low-quality trials (RR 0.79 , $95 \%$ CI $\left.0.66-0.94, P=0.007, I^{2}=0 \%\right)$, whereas trials with higher methodology scores did not show any significant effect (RR 1.06, $95 \%$ CI 0.95-1.19, $P=0.27$, heterogeneity $I^{2}=0 \%$ ). The overall tests for significance revealed statistically significant differences between these subgroups $(P=0.004)$ (see Additional file 3: Figure 54$)$. Neither high- nor low-quality trials demonstrated any effect on infections (test for subgroup differences $P=$ 0.72) (see Additional file 3: Figure S5).

\section{Sepsis vs. nonsepsis studies}

There was no effect of Se therapy on mortality in patients with sepsis (RR 0.92, $95 \%$ CI 0.79-1.07, $P=0.27$, $I^{2}=10 \%$ ) or without sepsis (RR 1.03, $95 \%$ CI 0.91-1.16, $P=0.68 ; I^{2}=0 \%$, test for subgroup differences $P=0.27$ ). However, there was a significant effect of parenteral Se on the reduction of infections in RCTs of patients 
Table 2 Reported outcomes of included randomized clinical trials evaluating intravenous selenium supplementation in critically ill patients

\begin{tabular}{|c|c|c|c|c|c|c|}
\hline \multirow[t]{2}{*}{ Study } & \multicolumn{2}{|l|}{ Mortality (\%) } & \multicolumn{2}{|l|}{ Infections (\%) } & \multicolumn{2}{|l|}{ LOS days } \\
\hline & Experimental & Control & Experimental & Control & Experimental & Control \\
\hline Kuklinski 1991 [38] & ICU 0/8 (0) & ICU 8/9 ( 89) & $N R$ & NR & NR & NR \\
\hline Zimmerman 1997 [39] & $3 / 20(15)$ & $8 / 20(40)$ & NR & NR & NR & NR \\
\hline \multirow[t]{4}{*}{ Berger 1998 [40] } & \multirow[t]{4}{*}{$1 / 10(10)$} & \multirow[t]{4}{*}{ 0/10 (0) } & $1.9 \pm 0.9(1-4)$ & $3.1 \pm 1.1(2-5)$ & ICU & ICU \\
\hline & & & \multirow[t]{3}{*}{ per patient } & \multirow[t]{3}{*}{ per patient } & $30 \pm 12(10)$ & $39 \pm 13(10)$ \\
\hline & & & & & Hospital & Hospital \\
\hline & & & & & $54 \pm 27(10)$ & $66 \pm 31(10)$ \\
\hline \multirow[t]{2}{*}{ Angstwurm 1999 [41] } & Hospital & Hospital & \multirow[t]{2}{*}{ NR } & \multirow[t]{2}{*}{$N R$} & \multirow[t]{2}{*}{$N R$} & \multirow[t]{2}{*}{$N R$} \\
\hline & 7/21 (33) & $11 / 21(52)$ & & & & \\
\hline \multirow[t]{4}{*}{ Porter 1999 [42] } & \multirow[t]{4}{*}{$0 / 9(0)$} & \multirow[t]{4}{*}{$0 / 9(0)$} & \multirow[t]{4}{*}{$5 / 9(56)$} & \multirow[t]{4}{*}{ 8/9 (89) } & $\mathrm{ICU}$ & $\mathrm{ICU}$ \\
\hline & & & & & $22 \pm 25.2$ & $35.8 \pm 21.9$ \\
\hline & & & & & Hospital & Hospital \\
\hline & & & & & $31.3 \pm 23.4$ & $49 \pm 30$ \\
\hline \multirow[t]{10}{*}{ Berger 2001 [43] } & (a) Selenium alone & \multirow[t]{10}{*}{$1 / 11(9)$} & (a) Selenium alone & \multirow[t]{10}{*}{$5 / 11(42)$} & (a) & $\mathrm{ICU}$ \\
\hline & $2 / 9(22)$ & & $5 / 9(56)$ & & $\mathrm{ICU}$ & $8.6 \pm 8.1(12)$ \\
\hline & \multirow{2}{*}{$\begin{array}{l}\text { (b) Selenium + zinc }+ \\
\text { a-tocopherol }\end{array}$} & & (b) Selenium + zinc + & & $8.0 \pm 4.0(9)$ & Hospital \\
\hline & & & & & Hospital & $64 \pm 39(12)$ \\
\hline & 0/11 (0) & & $3 / 11(27)$ & & $82 \pm 78(9)$ & \\
\hline & & & & & (b) & \\
\hline & & & & & $I C U$ & \\
\hline & & & & & $5.8 \pm 4.4(11)$ & \\
\hline & & & & & Hospital & \\
\hline & & & & & $60 \pm 48(11)$ & \\
\hline Linder 2004 [44] & Not specified & Not specified & NA & NA & Hospital & Hospital \\
\hline & $5 / 32(15.6)$ & 3/35 (8.6) & & & $24(9-44)$ & $26(11-46)$ \\
\hline Angstwurm 2007 [45] & 28-day & 28-day & New infections (HAP) & New infections (HAP) & $\mathrm{ICU}$ & ICU \\
\hline & $46 / 116(40)$ & $61 / 122(50)$ & 10/116 (9) & 10/122 (8) & $15.1 \pm 10(116)$ & $12.7 \pm 9(122)$ \\
\hline Berger 2007 [46] & 1/11 (9) & 1/10 (10) & $2.1 \pm 1.0$ & $3.6 \pm 1.3$ & $\mathrm{ICU}$ & $\mathrm{ICU}$ \\
\hline & & & per patient & per patient & $35 \pm 27(11)$ & $47 \pm 37(10)$ \\
\hline Forceville 2007 [47] & 28-day & 28-day & Superinfection & Superinfection & $\mathrm{ICU}$ & $\mathrm{ICU}$ \\
\hline & $14 / 31(45)$ & $13 / 29(45)$ & $1 / 31(3)$ & $2 / 29(7)$ & $21(7-40)$ & $18(10-31)$ \\
\hline & 6-month & 6-month & & & Hospital & Hospital \\
\hline & 18/31 (59) & $20 / 29(68)$ & & & $25(7-68)$ & $33(11-51)$ \\
\hline & 1-year & 1-year & & & & \\
\hline & $66 \%$ & $71 \%$ & & & & \\
\hline Mishra 2007 [48] & ICU 8/18 (44) & ICU 11/22 (61) & $1.5 \pm 1.9$ & $1.8 \pm 1.6$ & $\mathrm{ICU}$ & ICU \\
\hline & Hospital & Hospital & per patient & per patient & $21.3 \pm 16.2(18)$ & $20.8 \pm 21.8(18$ \\
\hline & $11 / 18(61)$ & 15/22 (68) & & & & \\
\hline & 28-day & 28-day & & & & \\
\hline & 8/18 (44) & $11 / 22(50)$ & & & & \\
\hline Berger 2008 [49] & ICU & ICU & $36 / 102(35)$ & $34 / 98(35)$ & $\mathrm{ICU}$ & $\mathrm{ICU}$ \\
\hline & 8/102 (8) & $5 / 98(5)$ & & & $5.8 \pm 5.4(102)$ & $5.4 \pm 5.7(98)$ \\
\hline & Hospital & Hospital & & & Hospital & Hospital \\
\hline & 14/102 (14) & 9/98 (11) & & & $23 \pm 20(102)$ & $26 \pm 20(98)$ \\
\hline
\end{tabular}


Table 2 Reported outcomes of included randomized clinical trials evaluating intravenous selenium supplementation in critically ill patients (Continued)

\begin{tabular}{|c|c|c|c|c|c|c|}
\hline & 3-month & 3-month & & & & \\
\hline & 14/102 (14) & $11 / 98(11)$ & & & & \\
\hline \multirow[t]{2}{*}{ El-Attar 2009 [50] } & $\mathrm{ICU}$ & $\mathrm{ICU}$ & VAP & VAP & $N R$ & NR \\
\hline & $2 / 40(5.6)$ & $1 / 40(2.9)$ & $5 / 36(14)$ & $7 / 34(21)$ & & \\
\hline \multirow[t]{2}{*}{ Montoya 2009 [51] } & Hospital & Hospital & NR & NR & Hospital & Hospital \\
\hline & 6/34 (18) & $8 / 34(24)$ & & & $12(12-14)$ & $17(14-20)$ \\
\hline \multirow[t]{4}{*}{ Andrews 2011 [52] } & $\mathrm{ICU}$ & ICU & Confirmed & Confirmed & $\mathrm{ICU}$ & $\mathrm{ICU}$ \\
\hline & $84 / 251(33)$ & $84 / 251(33)$ & $104 / 251(41)$ & $121 / 251(48)$ & $\begin{array}{l}13.2 \\
(\text { IQR } 7.8-23.7)\end{array}$ & $\begin{array}{l}15.1 \\
(\text { IQR } 8.3-28.4)\end{array}$ \\
\hline & 6-month & 6-month & & & Hospital & Hospital \\
\hline & 107/251 (43) & $114 / 251(45)$ & & & $\begin{array}{l}29.8 \\
(\text { IQR 14.7-52.4) }\end{array}$ & $\begin{array}{l}31.2 \\
(\text { IQR 15.1-57.8) }\end{array}$ \\
\hline \multirow[t]{4}{*}{ Manzanares 2011 [53] } & $\mathrm{ICU}$ & $\mathrm{ICU}$ & VAP & VAP & $\mathrm{ICU}$ & $\mathrm{ICU}$ \\
\hline & $3 / 15(20)$ & $5 / 16(31)$ & $3 / 15(20)$ & $7 / 16(44)$ & $14 \pm 11(15)$ & $13 \pm 6(16)$ \\
\hline & Hospital & Hospital & & & & \\
\hline & $5 / 15(33)$ & $7 / 16(44)$ & & & & \\
\hline \multirow[t]{2}{*}{ Valenta 2011 [54] } & 28-day & 28-day & NR & NR & $N R$ & $N R$ \\
\hline & $19 / 75(25)$ & 24/75 (32) & & & & \\
\hline \multirow[t]{10}{*}{ Heyland 2013 [16] } & Hospital & Hospital & All & All & $\mathrm{ICU}$ & ICU \\
\hline & 216/617 (35) & 199/601 (33) & 168/617 (27) & 181/601 (30) & $14.2 \pm 22.7(617)$ & $13.8 \pm 23.1(601)$ \\
\hline & 14-day & 14-day & VAP & VAP & Hospital & Hospital \\
\hline & $154 / 617(25)$ & $132 / 601(22)$ & $71 / 617(12)$ & 95/601 (16) & $31.2 \pm 50.2(617)$ & $29.5 \pm 44.8(601)$ \\
\hline & 28-day & 28-day & & & & \\
\hline & 190/617 (31) & 173/601 (29) & & & & \\
\hline & 3-month & 3-month & & & & \\
\hline & 239 & 222 & & & & \\
\hline & 6-month & 6-month & & & & \\
\hline & 250 & 235 & & & & \\
\hline \multirow[t]{6}{*}{ Woth 2014 [14] } & In 14-day study period & In 14-day study period & Gram-negative & Gram-negative & NR & $N R$ \\
\hline & 9/21 (43) & $11 / 19(58)$ & 8/21 (38) & $3 / 19(16)$ & & \\
\hline & & & Gram-positive & Gram-positive & & \\
\hline & & & $3 / 21(14)$ & 2/19 (11) & & \\
\hline & & & Fungal & Fungal & & \\
\hline & & & $1 / 21(5)$ & $0 / 19(0)$ & & \\
\hline \multirow[t]{5}{*}{ Bloos 2016 [17] } & 28-day & 28-day & \multirow{2}{*}{$\begin{array}{l}\text { Secondary infections, } \\
\text { day } 14\end{array}$} & \multirow{2}{*}{$\begin{array}{l}\text { Secondary infections, } \\
\text { day } 14\end{array}$} & ICU & $\mathrm{ICU}$ \\
\hline & $152 / 543(28)$ & $137 / 546(25)$ & & & $11(5-22)$ & $12(6-24)$ \\
\hline & 90-day & 90-day & $243 / 543$ (44.7 \%) & 269/546 (49.3 \%) & Hospital & Hospital \\
\hline & $198 / 543(38)$ & $201 / 546(38)$ & $\begin{array}{l}\text { Secondary infections, } \\
\text { day } 21\end{array}$ & $\begin{array}{l}\text { Secondary infections, } \\
\text { day } 21\end{array}$ & $26(16-42)$ & $29(17-50)$ \\
\hline & & & $319 / 543$ (58.8 \%) & $323 / 546$ (59.2 \%) & & \\
\hline \multirow[t]{4}{*}{ Chelkeba 2015 [15] } & 28-day & 28-day & VAP & VAP & $\mathrm{ICU}$ & $\mathrm{ICU}$ \\
\hline & $9 / 29(31)$ & $10 / 25(40)$ & $16 / 26(61.5)$ & $21 / 25(84)$ & $19.7 \pm 11(26)$ & $23.8 \pm 13(25)$ \\
\hline & & & & & Hospital & Hospital \\
\hline & & & & & $25.2 \pm 10(26)$ & $24.5 \pm 9(25)$ \\
\hline
\end{tabular}




\begin{tabular}{|c|c|c|c|c|c|c|c|c|c|c|c|}
\hline \multirow[b]{2}{*}{ Study or Subgroup } & \multicolumn{2}{|c|}{ Selenium } & \multicolumn{2}{|c|}{ Control } & \multicolumn{3}{|c|}{ Risk Ratio } & \multirow{2}{*}{\multicolumn{4}{|c|}{$\begin{array}{c}\text { Risk Ratio } \\
\text { M-H, Random, } 95 \% \mathrm{Cl}\end{array}$}} \\
\hline & Events & Total & Events & Total & Weight & M-H, Random, 95\% Cl & Year & & & & \\
\hline \multicolumn{10}{|c|}{ 4.3.1 PN Selenium Monotherapy } & & \\
\hline Kuklinski & 0 & 8 & 8 & 9 & $0.1 \%$ & $0.07[0.00,0.98]$ & 1991 & & & & \\
\hline Zimmerman & 3 & 20 & 8 & 20 & $0.6 \%$ & $0.38[0.12,1.21]$ & 1997 & & & & \\
\hline Angstwurm 1999 & 7 & 21 & 11 & 21 & $1.6 \%$ & $0.64[0.31,1.32]$ & 1999 & & & & \\
\hline Mishra & 11 & 18 & 15 & 22 & $3.9 \%$ & $0.90[0.56,1.43]$ & 2007 & & & & \\
\hline Forceville & 14 & 31 & 13 & 29 & $2.7 \%$ & $1.01[0.58,1.76]$ & 2007 & & $\longrightarrow$ & & \\
\hline Angstwurm 2007 & 46 & 116 & 61 & 122 & $10.4 \%$ & $0.79[0.60,1.06]$ & 2007 & & $\rightarrow$ & & \\
\hline Montoya & 6 & 34 & 8 & 34 & $0.9 \%$ & $0.75[0.29,1.93]$ & 2009 & & & & \\
\hline Andrews & 84 & 251 & 84 & 251 & $13.9 \%$ & $1.00[0.78,1.28]$ & 2011 & & & & \\
\hline Valenta & 19 & 75 & 24 & 75 & $3.3 \%$ & $0.79[0.48,1.32]$ & 2011 & & . & & \\
\hline Manzanares & 3 & 15 & 5 & 16 & $0.5 \%$ & $0.64[0.18,2.22]$ & 2011 & & & & \\
\hline Woth & 9 & 21 & 11 & 19 & $2.2 \%$ & $0.74[0.40,1.38]$ & 2014 & & & & \\
\hline Chelkeba & 9 & 29 & 10 & 25 & $1.6 \%$ & $0.78[0.38,1.60]$ & 2016 & & & & \\
\hline Bloos & 152 & 543 & 137 & 546 & $21.6 \%$ & $1.12[0.92,1.36]$ & 2016 & & & & \\
\hline Subtotal $(95 \% \mathrm{Cl})$ & & 1182 & & 1189 & $63.4 \%$ & $0.91[0.79,1.04]$ & & & & & \\
\hline Total events & 363 & & 395 & & & & & & & & \\
\hline \multicolumn{12}{|c|}{ Heterogeneity: $\mathrm{Tau}^{2}=0.01 ; \mathrm{Chi}^{2}=13.59, \mathrm{df}=12(\mathrm{P}=0.33) ; \mathrm{I}^{2}=12 \%$} \\
\hline \multicolumn{12}{|c|}{ Test for overall effect: $Z=1.42(P=0.16)$} \\
\hline \multicolumn{12}{|c|}{ 4.3.2 PN Selenium Combined } \\
\hline Berger 1998 & 1 & 10 & 0 & 10 & $0.1 \%$ & $3.00[0.14,65.90]$ & 1998 & & & & \\
\hline Porter & 0 & 9 & 0 & 9 & & Not estimable & 1999 & & & & \\
\hline Berger 2001b & 0 & 11 & 1 & 12 & $0.1 \%$ & $0.36[0.02,8.04]$ & 2001 & & & & \\
\hline Berger 2001a & 2 & 9 & 1 & 12 & $0.2 \%$ & $2.67[0.28,25.04]$ & 2001 & & & & \\
\hline Berger 2007 & 1 & 11 & 1 & 10 & $0.1 \%$ & $0.91[0.07,12.69]$ & 2007 & & & & \\
\hline Berger 2008 & 14 & 102 & 9 & 98 & $1.4 \%$ & $1.49[0.68,3.29]$ & 2008 & & & & \\
\hline El-Attar & 2 & 40 & 1 & 40 & $0.2 \%$ & $2.00[0.19,21.18]$ & 2009 & & & & \\
\hline Heyland & 216 & 617 & 199 & 601 & $34.6 \%$ & $1.06[0.90,1.24]$ & 2013 & & & & \\
\hline Subtotal $(95 \% \mathrm{Cl})$ & & 809 & & 792 & $36.6 \%$ & $1.08[0.93,1.25]$ & & & & & \\
\hline Total events & 236 & & 212 & & & & & & & & \\
\hline \multicolumn{12}{|c|}{ Heterogeneity: $\mathrm{Tau}^{2}=0.00 ; \mathrm{Chi}^{2}=2.53, \mathrm{df}=6(\mathrm{P}=0.86) ; \mathrm{I}^{2}=0 \%$} \\
\hline \multicolumn{12}{|c|}{ Test for overall effect: $Z=0.96(P=0.33)$} \\
\hline Total $(95 \% \mathrm{Cl})$ & & 1991 & & 1981 & $100.0 \%$ & $0.98[0.90,1.08]$ & & & 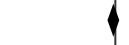 & & \\
\hline Total events & 599 & & 607 & & & & & & & & \\
\hline \multicolumn{8}{|c|}{ Heterogeneity: $\mathrm{Tau}^{2}=0.00 ; \mathrm{Chi}^{2}=18.31, \mathrm{df}=19(\mathrm{P}=0.50) ; \mathrm{I}^{2}=0 \%$} & 0.1 & 1 & 10 & 100 \\
\hline \multicolumn{8}{|c|}{ Test for overall effect: $Z=0.36(P=0.72)$} & Favours exp & erimental & Favours control & \\
\hline \multicolumn{12}{|c|}{ Test for subgroup differences: $\mathrm{Chi}^{2}=2.77, \mathrm{df}=1(\mathrm{P}=0.10), \mathrm{I}^{2}=63.9 \%$} \\
\hline
\end{tabular}

without sepsis (RR 0.88, $95 \%$ CI $0.78-0.99, P=0.03$, $\left.I^{2}=0 \%\right)$ compared with trials of patients with sepsis (RR 0.99, $95 \%$ CI 0.90-1.10, $P=0.90, I^{2}=0 \%$, test for subgroup differences $P=0.12$ ) (Fig. 5).

\section{Effect of geographic representation of study patients on outcomes}

We found no significant treatment effect differences in mortality (test for subgroup differences $P=0.33$ ) and infections (test for subgroup differences $P=0.57$ ) when we compared studies in which researchers administered IV $\mathrm{Se}$ in North America vs. other geographic regions (see Additional file 4: Figs. S6 and S7).

\section{Publication bias}

There was no indication that publication bias influenced the observed aggregated results. In fact, funnel plots were created for each study outcome, and the tests of asymmetry showed a trend for mortality $(P=0.08)$, although the data were not significant for overall infections $(P=0.19)$, hospital LOS $(P=0.50)$, or MV days $(P=0.37)$. However, the test for asymmetry was significant for ICU LOS $(P=0.047)$.

\section{Discussion}

This updated systematic review and meta-analysis of the effects of parenteral Se as single or combined therapy on 


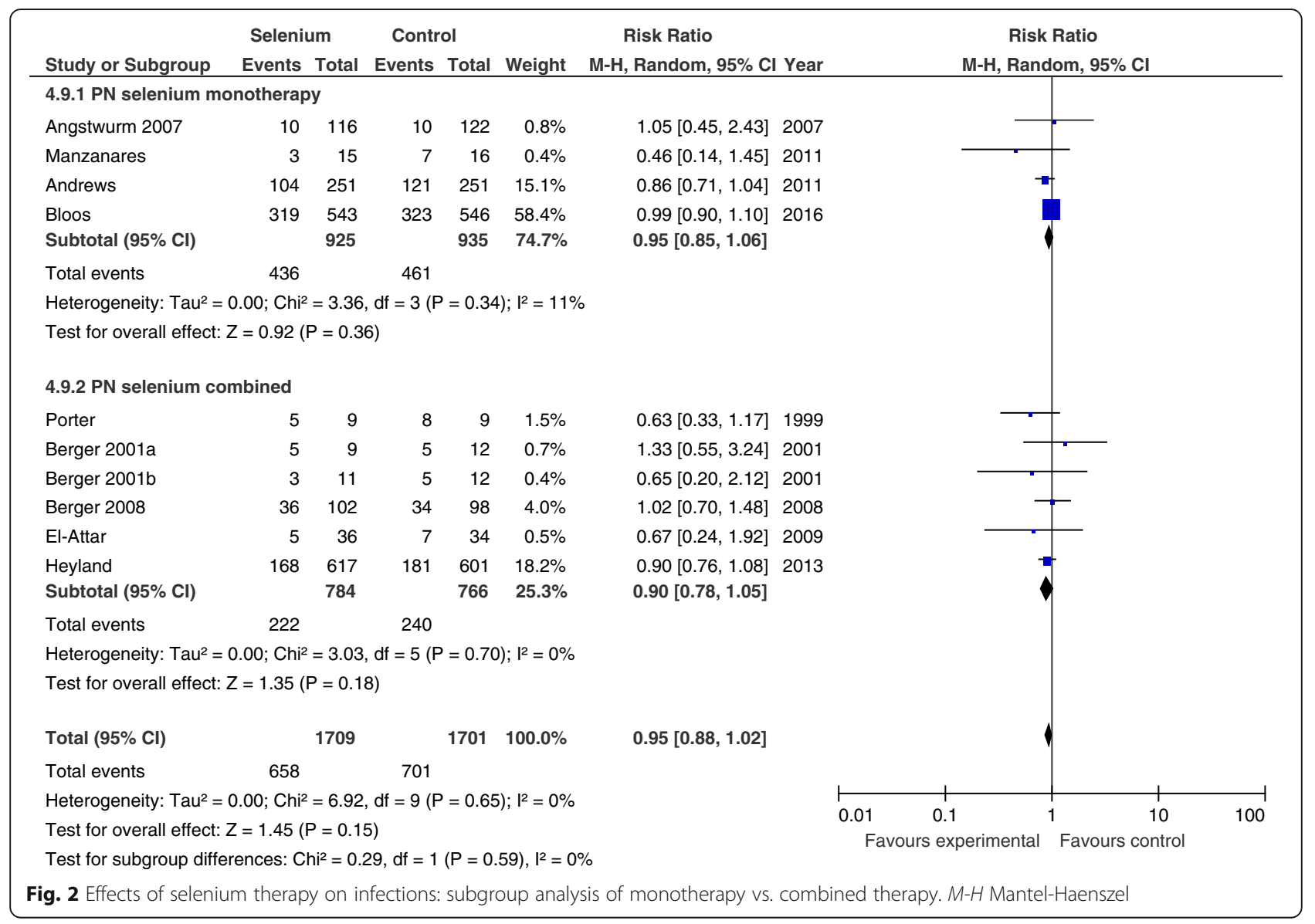

clinical outcomes included 21 trials with a total of 4044 critically ill patients. Including the two largest recent RCTs [16, 17], the main finding of our meta-analysis is that there was a lack of treatment effect when critically ill patients were treated with IV Se as single or combined therapy. In fact, we were unable to demonstrate any effect of IV Se supplementation on mortality or any significant effect on infections, ventilator days, or ICU and hospital LOS. Furthermore, our a priori defined subgroup analyses did not show any treatment effects on mortality. However, we found a significant effect of Se therapy on infectious complications in those studies without an initial IV loading dose, as well as a similar effect in trials conducted in nonseptic patients.

Over the last few years, different meta-analyses of Se supplementation in the critically ill have been published [8-13]. The differences with our present review are due largely to the variations in the studies included in the reviews, as our systematic review and meta-analysis is the first to include the two largest trials done to date on Se therapy in the critically ill.

Our present findings do not support the concept of pharmaconutrition by which micronutrients such as Se are provided in high (i.e., supraphysiological) doses in order to derive a pharmacological effect. Conversely to previous findings regarding IV high-dose Se monotherapy $[8,9]$, we did not find an overall effect of Se on infectious complications in the critically ill, although parenteral Se without an initial bolus significantly reduced infections. Nonetheless, the overall point estimate on infections of Se monotherapy is primarily and largely influenced by the Bloos et al. [17] study, the largest trial $(n=1089)(n=1089)$ on pharmaconutrition with high-dose Se monotherapy and PCT-guided antibiotic therapy in patients with severe sepsis and septic shock. After giving an initial IV loading dose of $1000 \mu \mathrm{g}$ sodium selenite followed by a continuous infusion of $1000 \mu$ g sodium selenite daily for no longer than 21 days, Bloos et al. [17] found that secondary infections were similar in both groups of patients. Interestingly enough, the SISPCT study [17] demonstrated that high-dose Se supplementation had no therapeutic benefit in septic patients, although plasma Se depletion at baseline was restored to the normal range already by treatment day 1 , suggesting that correction of plasma Se concentration may have no beneficial value. According to this study, plasma Se levels in the Se or placebo groups were not affected by allocation to the PCT guidance or non-PCT 


\begin{tabular}{|c|c|c|c|c|c|c|c|c|c|c|}
\hline \multirow[b]{2}{*}{ Study or Subgroup } & \multicolumn{2}{|c|}{ Selenium } & \multicolumn{2}{|c|}{ Control } & \multicolumn{3}{|c|}{ Risk Ratio } & \multirow{2}{*}{\multicolumn{3}{|c|}{$\begin{array}{c}\text { Risk Ratio } \\
\text { M-H, Random, } 95 \% \text { Cl }\end{array}$}} \\
\hline & Events & Total & Events & Total & Weight & M-H, Random, 95\% Cl & Year & & & \\
\hline \multicolumn{11}{|c|}{ 4.4.1 PN Selenium loading dose } \\
\hline Zimmerman & 3 & 20 & 8 & 20 & $0.6 \%$ & $0.38[0.12,1.21]$ & 1997 & & & \\
\hline Angstwurm 2007 & 46 & 116 & 61 & 122 & $10.4 \%$ & $0.79[0.60,1.06]$ & 2007 & & $\rightarrow$ & \\
\hline Berger 2008 & 14 & 102 & 9 & 98 & $1.4 \%$ & $1.49[0.68,3.29]$ & 2008 & & & \\
\hline Montoya & 6 & 34 & 8 & 34 & $0.9 \%$ & $0.75[0.29,1.93]$ & 2009 & & & \\
\hline Valenta & 19 & 75 & 24 & 75 & $3.3 \%$ & $0.79[0.48,1.32]$ & 2011 & & & \\
\hline Manzanares & 3 & 15 & 5 & 16 & $0.5 \%$ & $0.64[0.18,2.22]$ & 2011 & & & \\
\hline Woth & 9 & 21 & 11 & 19 & $2.2 \%$ & $0.74[0.40,1.38]$ & 2014 & & E & \\
\hline Chelkeba & 9 & 29 & 10 & 25 & $1.6 \%$ & $0.78[0.38,1.60]$ & 2016 & & $\sqrt{7}$ & \\
\hline Bloos & 152 & 543 & 137 & 546 & $21.6 \%$ & $1.12[0.92,1.36]$ & 2016 & & & \\
\hline Subtotal $(95 \% \mathrm{Cl})$ & & 955 & & 955 & $42.5 \%$ & $0.90[0.75,1.08]$ & & & & \\
\hline Total events & 261 & & 273 & & & & & & & \\
\hline \multicolumn{11}{|c|}{ Heterogeneity: $\mathrm{Tau}^{2}=0.01 ; \mathrm{Chi}^{2}=9.80, \mathrm{df}=8(\mathrm{P}=0.28) ; \mathrm{I}^{2}=18 \%$} \\
\hline \multicolumn{11}{|c|}{ Test for overall effect: $Z=1.11(P=0.27)$} \\
\hline \multicolumn{11}{|c|}{ 4.4.2 PN selenium no loading dose } \\
\hline Kuklinski & 0 & 8 & 8 & 9 & $0.1 \%$ & $0.07[0.00,0.98]$ & 1991 & $\longleftarrow$ & & \\
\hline Berger 1998 & 1 & 10 & 0 & 10 & $0.1 \%$ & $3.00[0.14,65.90]$ & 1998 & & & \\
\hline Angstwurm 1999 & 7 & 21 & 11 & 21 & $1.6 \%$ & $0.64[0.31,1.32]$ & 1999 & & & \\
\hline Porter & 0 & 9 & 0 & 9 & & Not estimable & 1999 & & & \\
\hline Berger 2001a & 2 & 9 & 1 & 12 & $0.2 \%$ & $2.67[0.28,25.04]$ & 2001 & & & \\
\hline Berger 2001b & 0 & 11 & 1 & 12 & $0.1 \%$ & $0.36[0.02,8.04]$ & 2001 & & & \\
\hline Mishra & 11 & 18 & 15 & 22 & $3.9 \%$ & $0.90[0.56,1.43]$ & 2007 & & & \\
\hline Forceville & 14 & 31 & 13 & 29 & $2.7 \%$ & $1.01[0.58,1.76]$ & 2007 & & & \\
\hline Berger 2007 & 1 & 11 & 1 & 10 & $0.1 \%$ & $0.91[0.07,12.69]$ & 2007 & & & \\
\hline El-Attar & 2 & 40 & 1 & 40 & $0.2 \%$ & $2.00[0.19,21.18]$ & 2009 & & & \\
\hline Andrews & 84 & 251 & 84 & 251 & $13.9 \%$ & $1.00[0.78,1.28]$ & 2011 & & $r$ & \\
\hline Heyland & 216 & 617 & 199 & 601 & $34.6 \%$ & $1.06[0.90,1.24]$ & 2013 & & & \\
\hline Subtotal $(95 \% \mathrm{Cl})$ & & 1036 & & 1026 & $57.5 \%$ & $1.01[0.90,1.14]$ & & & & \\
\hline Total events & 338 & & 334 & & & & & & & \\
\hline \multicolumn{11}{|c|}{ Heterogeneity: $\mathrm{Tau}^{2}=0.00 ; \mathrm{Chi}^{2}=8.03, \mathrm{df}=10(\mathrm{P}=0.63) ; \mathrm{I}^{2}=0 \%$} \\
\hline \multicolumn{11}{|c|}{ Test for overall effect: $Z=0.21(P=0.83)$} \\
\hline Total $(95 \% \mathrm{Cl})$ & & 1991 & & 1981 & $100.0 \%$ & $0.98[0.90,1.08]$ & & & & \\
\hline Total events & 599 & & 607 & & & & & & & \\
\hline \multicolumn{8}{|c|}{ Heterogeneity: $\mathrm{Tau}^{2}=0.00 ; \mathrm{Chi}^{2}=18.31, \mathrm{df}=19(\mathrm{P}=0.50) ; \mathrm{I}^{2}=0 \%$} & 0.01 & 10 & 100 \\
\hline \multicolumn{8}{|c|}{ Test for overall effect: $Z=0.36(P=0.72)$} & Favours exp & perimental Favours control & \\
\hline \multicolumn{11}{|c|}{ Test for subgroup differences: $\mathrm{Chi}^{2}=1.10, \mathrm{df}=1(\mathrm{P}=0.30), \mathrm{I}^{2}=8.8 \%$} \\
\hline
\end{tabular}

guidance group. Our sensitivity analysis showed that, after excluding the PCT guidance arm, the new RR was 0.95 (previous RR 0.98), which confirms that excluding the PCT arm of the Bloos et al. study [17] did not affect the overall result of our analysis.

In contrast to previous knowledge, we were unable to find beneficial effects with daily doses higher than $500 \mu \mathrm{g}$ or with providing an initial loading dose as an IV bolus (usually $1000-2000 \mu \mathrm{g}$ in 30 minutes to $2 \mathrm{~h}$ ). However, the absence of a significant test of subgroup differences weakens any inferences drawn from this subgroup analysis, but it likely shows the ineffectiveness of employing a loading dose with an aim of improving outcomes. So far, it has been proposed that a loading dose of 1000-2000 $\mu \mathrm{g}$ Se as pentahydrate sodium selenite has prooxidant and cytotoxic effects $[1,53,55]$. In a previous pharmacokinetic study [31], it was demonstrated that an initial IV bolus of $2000 \mu \mathrm{g}$ followed by a continuous infusion of $1600 \mu \mathrm{g} /$ day was the most effective dose for returning serum Se to physiologic levels and safely maximizing extracellular GPx activity and therefore the antioxidant capacity in critically ill patients. According to current knowledge, a very high Se concentration may be able to produce an inhibition of nuclear factor- $\mathrm{kB}$ binding to DNA, controlling gene expression and the synthesis of proinflammatory cytokines [56, 57], and also may be able to induce apoptosis and cytotoxicity in activated proinflammatory cells [58]. In addition, 


\begin{tabular}{|c|c|c|c|c|c|c|c|c|c|c|c|}
\hline \multirow[b]{2}{*}{ Study or Subgroup } & \multicolumn{2}{|c|}{ Selenium } & \multicolumn{2}{|c|}{ Control } & \multicolumn{3}{|c|}{ Risk Ratio } & \multirow{2}{*}{\multicolumn{4}{|c|}{$\begin{array}{c}\text { Risk Ratio } \\
\text { M-H, Random, } 95 \% \mathrm{Cl}\end{array}$}} \\
\hline & Events & Total & Events & Total & Weight & M-H, Random, 95\% Cl & Year & & & & \\
\hline \multicolumn{12}{|c|}{ 4.10.1 PN selenium loading dose } \\
\hline Angstwurm 2007 & 10 & 116 & 10 & 122 & $0.8 \%$ & $1.05[0.45,2.43]$ & 2007 & & & & \\
\hline Berger 2008 & 36 & 102 & 34 & 98 & $4.0 \%$ & $1.02[0.70,1.48]$ & 2008 & & & & \\
\hline Manzanares & 3 & 15 & 7 & 16 & $0.4 \%$ & $0.46[0.14,1.45]$ & 2011 & & & & \\
\hline Bloos & 319 & 543 & 323 & 546 & $58.4 \%$ & $0.99[0.90,1.10]$ & 2016 & & & & \\
\hline Subtotal $(95 \% \mathrm{Cl})$ & & 776 & & 782 & $63.6 \%$ & $0.99[0.90,1.09]$ & & & & & \\
\hline Total events & 368 & & 374 & & & & & & & & \\
\hline \multicolumn{12}{|c|}{ Heterogeneity: $\mathrm{Tau}^{2}=0.00 ; \mathrm{Chi}^{2}=1.77, \mathrm{df}=3(\mathrm{P}=0.62) ; \mathrm{I}^{2}=0 \%$} \\
\hline \multicolumn{12}{|c|}{ Test for overall effect: $Z=0.21(P=0.84)$} \\
\hline \multicolumn{12}{|c|}{ 4.10.2 PN selenium no loading dose } \\
\hline Porter & 5 & 9 & 8 & 9 & $1.5 \%$ & $0.63[0.33,1.17]$ & 1999 & & & & \\
\hline Berger 2001b & 3 & 11 & 5 & 12 & $0.4 \%$ & $0.65[0.20,2.12]$ & 2001 & & & & \\
\hline Berger 2001a & 5 & 9 & 5 & 12 & $0.7 \%$ & $1.33[0.55,3.24]$ & 2001 & & & & \\
\hline El-Attar & 5 & 36 & 7 & 34 & $0.5 \%$ & $0.67[0.24,1.92]$ & 2009 & & 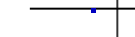 & & \\
\hline Andrews & 104 & 251 & 121 & 251 & $15.1 \%$ & $0.86[0.71,1.04]$ & 2011 & & 一町 & & \\
\hline Heyland & 168 & 617 & 181 & 601 & $18.2 \%$ & $0.90[0.76,1.08]$ & 2013 & & & & \\
\hline Subtotal $(95 \% \mathrm{Cl})$ & & 933 & & 919 & $36.4 \%$ & $0.87[0.77,0.99]$ & & & & & \\
\hline Total events & 290 & & 327 & & & & & & & & \\
\hline \multicolumn{12}{|c|}{ Heterogeneity: $\mathrm{Tau}^{2}=0.00 ; \mathrm{Chi}^{2}=2.61, \mathrm{df}=5(\mathrm{P}=0.76) ; \mathrm{I}^{2}=0 \%$} \\
\hline \multicolumn{12}{|c|}{ Test for overall effect: $Z=2.13(P=0.03)$} \\
\hline Total $(95 \% \mathrm{Cl})$ & & 1709 & & 1701 & $100.0 \%$ & $0.95[0.88,1.02]$ & & & & & \\
\hline Total events & 658 & & 701 & & & & & & & & \\
\hline \multicolumn{8}{|c|}{ Heterogeneity: $\mathrm{Tau}^{2}=0.00 ; \mathrm{Chi}^{2}=6.92, \mathrm{df}=9(\mathrm{P}=0.65) ; \mathrm{I}^{2}=0 \%$} & 0.01 & 1 & 10 & 100 \\
\hline \multicolumn{8}{|c|}{ Test for overall effect: $Z=1.45(P=0.15)$} & Favours exp & erimental $F$ & Eavours control & \\
\hline \multicolumn{12}{|c|}{ Test for subgroup differences: $\mathrm{Chi}^{2}=2.49, \mathrm{df}=1(\mathrm{P}=0.11), \mathrm{I}^{2}=59.8 \%$} \\
\hline \multicolumn{12}{|c|}{ Fig. 4 Effects of Selenium therapy on infections: subgroup analysis of loading dose vs. none. M-H Mantel-Haenszel } \\
\hline
\end{tabular}

using an experimental model of sepsis, Wang et al. [59] demonstrated that an IV Se bolus improved hemodynamic status, decreased inflammation biomarkers, and reduced mortality. Meanwhile, contrary to our present data, in 2012 we found that a parenteral loading dose showed a trend toward reduction in mortality, whereas studies that did not use a bolus-loading dose did not show any effect on mortality. Similarly, Huang et al. [9], after aggregating nine RCTs on Se monotherapy, demonstrated that an IV bolus was associated with a significant reduction in mortality (RR 0.73, $95 \% \mathrm{CI}$ $0.58-0.94, P=0.01$ ). However, neither of the previous meta-analyses considered the SISPCT study [17].

So far, most clinical studies using Se at low doses have been underpowered and have involved Se administered in a cocktail approach. Thus, positive results in those trials cannot be clearly attributed solely to Se supplementation. Notwithstanding this, according to the concept of nutrient replacement, by which micronutrient substitution is aimed at replenishing losses and target restoration of physiological function [60], Se must be supplemented at standard doses by the enteral $(77-100 \mu \mathrm{g} /$ day $)$ or the parenteral (100$400 \mu \mathrm{g} /$ day) route [61] because the results of our meta-analysis do not refute previously recommended Se substitution doses.

Despite earlier results of the Angstwurm study [41], which demonstrated that IV high-dose Se substitution in septic patients with an Acute Physiology and Chronic Health Evaluation II score higher than 15 significantly reduced the requirements of renal replacement therapy $(P=0.035)$, the post hoc analysis of the REDOXS [16] study demonstrated that patients with renal failure might have a worse outcome when treated with highdose antioxidants. In fact, Heyland and coworkers [16] demonstrated that both glutamine and antioxidants appeared harmful in patients with baseline renal dysfunction, showing a higher 28-day mortality (OR 3.39, $95 \%$ CI 1.41-8.17, and 3.07, $95 \%$ CI 1.24-7.59, for antioxidants alone and glutamine plus antioxidants, respectively). Nonetheless, in the recently published SISPCT study [17], researchers did not find any risk of increased harm in patients with baseline renal failure. In addition, in the present study, we were unable to find any deleterious effect of Se therapy on renal function in the critically ill.

Thus, the current understanding of why there is a lack of therapeutic effect of IV Se therapy in critically ill patients and patients with severe sepsis remains unclear. 


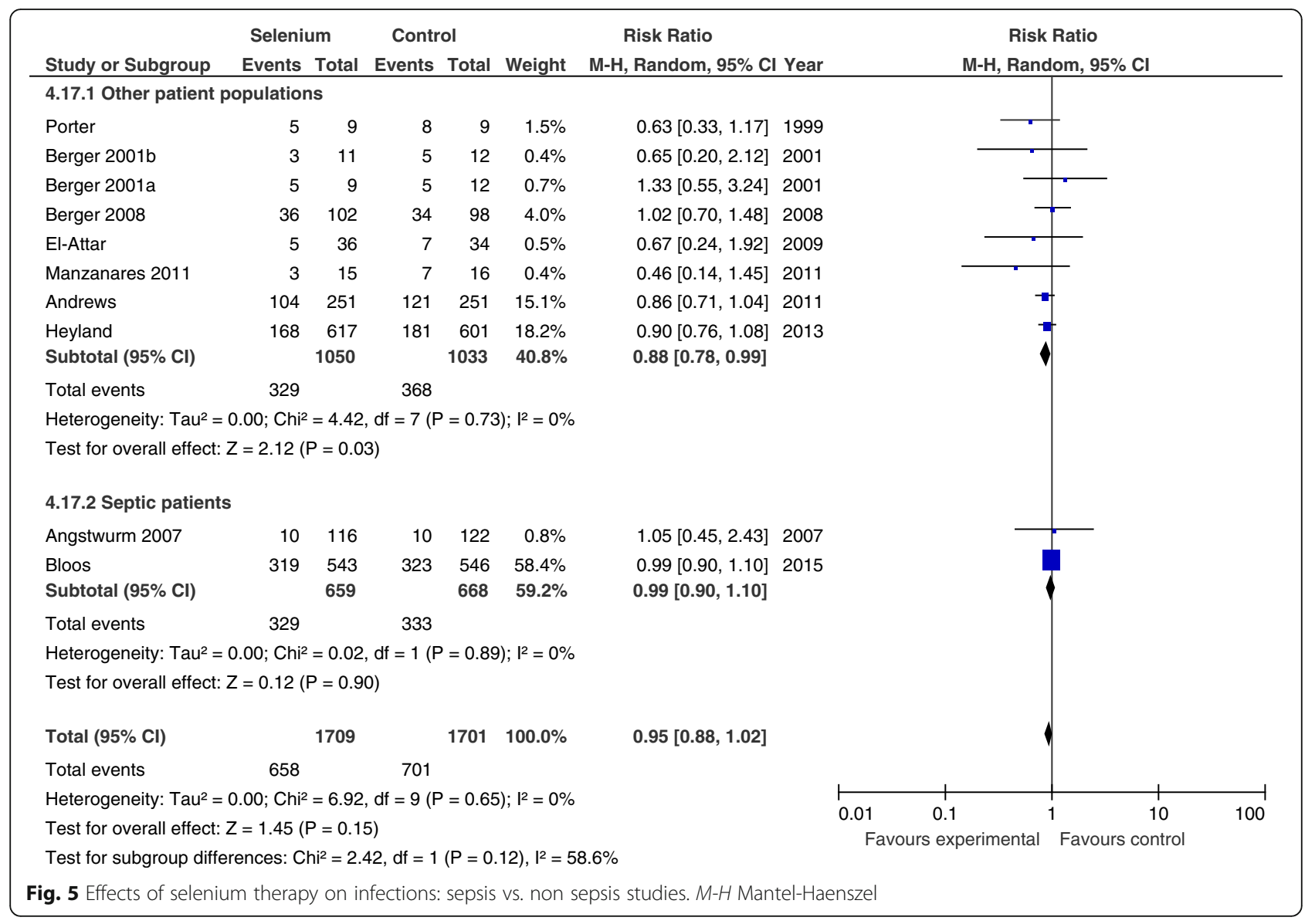

Notwithstanding this, Se therapy could show benefits in other patient populations that were not considered in our meta-analysis. In fact, it is currently known that circulating Se levels significantly decrease in the perioperative period of cardiac surgery [62]. Also, in a nonrandomized interventional trial, Stoppe et al. [63] demonstrated that high-dose sodium selenite therapy as a pharmaconutrient strategy was effective in preventing the decrease of Se levels and that clinical outcomes may be superior in supplemented patients compared with a historical control group. The SodiUm SeleniTe Administration IN Cardiac Surgery (SUSTAIN CSX trial, ClinicalTrials.gov identifier NCT02002247), an RCT aimed at evaluating the effects of perioperative high-dose Se supplementation in high-risk cardiac surgical patients undergoing complicated open heart surgery, is currently recruiting participants [64].

According to current evidence derived from recent trials and our meta-analysis, the updated version of Canadian Clinical Guidelines [65] recommended not using IV Se alone or in combination with other antioxidants in critically ill patients, which means that this strategy has recently been downgraded.
The strength of our meta-analysis is based on the fact that we used several methods to reduce bias (comprehensive literature search, duplicate data abstraction, comprehensive search strategy using specific criteria, and including non-English-language articles), we contacted trial authors to obtain additional data and refine our analysis, and we ultimately focused on clinically important primary outcomes in ICU patients. In addition, given the wide variety of clinical diagnoses and the heterogeneous population of ICU patients included in this systematic review (sepsis, septic shock, trauma, pancreatitis, surgical ICU patients), the results and conclusions may be applied to a broad and heterogeneous group of critically ill patients. While having a fairly large overall sample size and low heterogeneity, which makes the estimate quite robust, our subgroup analyses are limited by the small number of trials.

\section{Conclusions}

In this updated systematic review and meta-analysis, we found that parenteral Se as single or combined therapy with other antioxidant micronutrients had no effect on mortality, infections, renal function, ICU and hospital 
LOS, or ventilator days. Moreover, subgroup analyses did not show any treatment effects on mortality, although a significant effect on infections was found in those studies performed in nonseptic patients and when high-dose Se as an initial IV bolus was not administered.

According to our findings, IV Se therapy cannot be recommended for routine clinical use in critically ill patients. In view of these results, we suggest that we need to go back to basics and obtain more pharmacokinetic and pharmacodynamic data in specific patient populations with specific dosing strategies. We strongly believe that, without this first step exploring pharmacokinetic data, no further research on parenteral Se monotherapy in critically ill patients is warranted.

\section{Key messages}

- Se is an essential trace element with antioxidant, immunomodulatory, and anti-inflammatory effects that has been considered the cornerstone of the antioxidant defense system.

- Recently, in the largest trial on IV Se monotherapy, investigators were unable to find any clinical benefit of high-dose sodium selenite in patients with sepsis and septic shock.

- According to our findings, there is no evidence for a beneficial effect on mortality, infections, and other relevant clinical outcomes of high-dose IV Se as single or combined therapy (antioxidant cocktails) in critically ill patients.

- IV Se may be able to significantly reduce infections in those studies performed with nonseptic patients and when high-dose Se as an initial IV bolus is not administered.

\section{Additional files}

Additional file 1: Figure S1. Effect of Se on mortality. Sensitivity analysis after excluding the PCT guidance group in the SISPCT study. (DOCX $82 \mathrm{~kb}$ )

Additional file 2: Figure S2. Effect of IV Se on mortality: high- vs. low-mortality trials. Figure S3. Effect of IV Se on infections: high- vs. low-mortality trials. (DOCX $189 \mathrm{~kb}$ )

Additional file 3: Figure S4. Effect of IV Se on mortality: higher- vs. lower-quality trials. Figure S5. Effect of IV Se on infections: higher- vs. lower-quality trials. (DOCX $188 \mathrm{~kb}$ )

Additional file 4: Figure S6. Effect of geographic representation of study patients on outcomes: mortality. Figure S7. Effect of geographic representation of study patients on outcomes: infections. (DOCX $190 \mathrm{~kb}$ )

\section{Abbreviations}

APACHE: Acute Physiology and Chronic Health Evaluation; C.Random: Concealed randomization; COPD: Chronic obstructive pulmonary disease; D5W: Dextrose $5 \%$ in water; EN: Enteral nutrition; GPx: Glutathione peroxidase; HAP: Hospitalacquired pneumonia; Hosp: Hospital; ICU: Intensive care unit; ITT: Intention to treat; IV: Intravenous; LOS: Length of stay; M-H: Mantel-Haenszel; MV: Mechanical ventilation; NA: Nonattributable; NR: Nonreported; PN: Parenteral nutrition;
RCT: Randomized clinical trial; REDOXS: REducing Deaths due to Oxidative Stress trial; RevMan: Review Manager software; Se: Selenium; SIRS: Systemic inflammatory response syndrome; SISPCT: Sodium Selenite and Procalcitonin Guided Antimicrobial Therapy in Severe Sepsis study; SUSTAIN CSX ${ }^{\bullet}$ : SodiUm SeleniTe Administration IN Cardiac Surgery trial; TBSA: Total body surface area; VAP: Ventilator-associated pneumonia; WMD: Weighted mean difference

\section{Acknowledgements}

None.

\section{Funding}

No funding for the development, writing or submission of this manuscript was received.

\section{Availability of supporting data}

Not applicable.

\section{Authors' contributions}

WM contributed to development of the manuscript concept, study grading study selection, evaluation, and interpretation of data. WM also was primary author and editor of all drafts of the manuscript. ML contributed to study grading, selection, evaluation, and interpretation of data. ML performed much of the primary statistical analysis, meta-analysis, and data analysis and also contributed to the writing of the manuscript. GE contributed to study selection as well as evaluation and interpretation of data. GE also contributed substantially to the writing of the manuscript. PLL contributed to the development of study grading, study selection, and evaluation and interpretation of data. PLL also contributed to the writing of the manuscript. FB contributed to the development of study grading, study selection, and evaluation and interpretation of data. FB also contributed to the writing of the manuscript. DKH contributed to the development of the manuscript concept and to evaluation and interpretation of data. DKH also authored and edited all drafts of the manuscript. All authors read and approved the final manuscript.

\section{Competing interests}

WM received speaking honorarium from biosyn Arzneimittel, Fellbach, Germany. GE received lecture fees and travel support from Fresenius Kabi, Abbott, and B. Braun Melsungen and was a member of the Reducing Deaths Due to Oxidative Stress posttrial advisory board meeting (Fresenius Kabi) and gastrointestinal tolerance advisory board meeting (Nutricia). PLL received lectures fees from Fresenius Kabi. FB received lecture honoraria from biosyn Arzneimittel, Gilead Sciences, and CSL Behring. DKH received a research grant from biosyn Arzneimittel.

Consent for publication

Not applicable.

Ethics approval and consent to participate

Not applicable.

\section{Author details}

${ }^{1}$ Department of Critical Care, Intensive Care Unit, Hospital de Clínicas (University Hospital), Faculty of Medicine, Universidad de la República (UdelaR), Avenida Italia, 14th Floor, Montevideo 11.600, Uruguay. ${ }^{2}$ Clinical Evaluation Research Unit, Kingston General Hospital, Kingston, ON, Canada. ${ }^{3}$ Department of Anesthesiology and Intensive Care Medicine, University Medical Center Schleswig-Holstein, Campus Kiel, Kiel, Germany. ${ }^{4}$ Centre Hospitalier Universitaire de Sherbrooke, Hospital Fleurimont, Sherbrooke, QC, Canada. ${ }^{5}$ Department of Anesthesiology and Intensive Care Medicine, Jena University Hospital, Jena, Germany. ${ }^{6}$ Department of Medicine, Queen's

University, Kingston, ON, Canada.

Received: 26 July 2016 Accepted: 14 October 2016

Published online: 28 October 2016

\section{References}

1. Hardy G, Hardy I, Manzanares W. Selenium supplementation in the critically ill. Nutr Clin Pract. 2012;27:21-33.

2. Fairweather-Tait SJ, Bao Y, Broadley MR, Collings R, Ford D, Hesketh JE, et al. Selenium in human health and disease. Antioxid Redox Signal. 2011;14: 1337-83. 
3. Duntas LH, Benvenga S. Selenium: an element for life. Endocrine. 2015;48: 756-75.

4. Roman M, Jitaru P, Barbante C. Selenium biochemistry and its role for human health. Metallomics. 2014;6:25-54.

5. Manzanares W, Biestro A, Galusso F, Torre MH, Mañay N, Pittini G, et al. Serum selenium and glutathione peroxidase-3 activity: biomarkers of systemic inflammation in the critically ill. Intensive Care Med. 2009;35:882-9.

6. Forceville X, Vitoux D, Gauzit R, Combes A, Lahilaire P, Chappuis P. Selenium, systemic immune response syndrome, sepsis and outcome in critically ill patients. Crit Care Med. 1998;26:1536-44.

7. Heyland DK, Dhaliwal R, Suchner U, Berger MM. Antioxidants nutrients: a systematic review of trace elements and vitamins in the critically ill patient. Intensive Care Med. 2005;31:327-37.

8. Alhazzani W, Jacobi J, Sindi A, Hartog C, Reinhart K, Kokkoris S, et al. The effect of selenium therapy on mortality in patients with sepsis syndrome: a systematic review and meta-analysis of randomized controlled trials. Crit Care Med. 2013:41:1555-64.

9. Huang TS, Shyu YC, Chen HY, Lin LM, Lo CY, Yuan SS, et al. Effect of parenteral selenium supplementation in critically ill patients: a systematic review and meta-analysis. PLoS One. 2013;8, e54431.

10. Landucci F, Mancinelli P, De Gaudio AR, Virgili G. Selenium supplementation in critically ill patients: a systematic review and meta-analysis. J Crit Care. 2014:29:150-6.

11. Kong Z, Wang F, Ji S, Deng X, Xia Z. Selenium supplementation for sepsis: a meta-analysis of randomized controlled trials. Am J Emerg Med. 2013;31: $1170-5$

12. Allingstrup $M$, Afshari A. Selenium supplementation for critically ill adults. Cochrane Database Syst Rev. 2015;7, CD003703.

13. Manzanares W, Dhaliwal R, Jiang X, Heyland DK. Antioxidant micronutrients in the critically ill: a systematic review and meta-analysis. Crit Care. 2012;16:R66.

14. Woth G, Nagy B, Mérei Á, Ernyey B, Vincze R, Kaurics Z, et al. The effect of Na-selenite treatment on the oxidative stress-antioxidants balance of multiple organ failure. J Crit Care. 2014;29:883.e7-11.

15. Chelkeba L, Ahmadi A, Abdollahi M, Najafi A, Ghadimi MH, Mosaed R, et al. The effect of parenteral selenium on outcomes of mechanically ventilated patients following sepsis: a prospective randomized clinical trial. Ann Intensive Care. 2015:5:29.

16. Heyland DK, Muscedere J, Wischmeyer P, Cook D, Jones D, Albert M, et al. Canadian Critical Care Trials Group. A randomized trial of glutamine and antioxidants in critically ill patients. N Engl J Med. 2013;368:1489-97. A published erratum appears in N Engl J Med. 2013;368:1853.

17. Bloos F, Trips E, Nierhaus A, Briegel J, Heyland DK, Jaschinski U, et al. Effect of sodium selenite administration and procalcitonin-guided therapy on mortality in patients with severe sepsis: a randomized clinical trial. JAMA Intern Med. 2016;176:1266-76. doi:10.1001/jamainternmed.2016.2514.

18. Heyland DK, MacDonald S, Keefe L, Drover JW. Total parenteral nutrition in the critically ill patient: a meta-analysis. JAMA. 1998;280:2013-9.

19. DerSimonian R, Laird N. Meta-analysis in clinical trials. Control Clin Trials. 1986:7:177-88

20. Deeks JJ, Higgins JPT; Statistical Methods Group of The Cochrane Collaboration. Statistical algorithms in Review Manager 5. August 2010. http://ims.cochrane.org/revman/documentation/Statistical-methods-inRevMan-5.pdf. Accessed 15 Mar 2016

21. Egger M, Davey Smith G, Schneider M, Minder C. Bias in meta-analysis detected by a simple, graphical test. BMJ. 1997;315:629-34

22. Uden S, Bilton D, Nathan L, Hunt LP, Mains C, Braganza JM. Antioxidant therapy for recurrent pancreatitis: placebo-controlled trial. Aliment Pharmacol Ther. 1990;4:357-71.

23. Uden S, Schofield D, Miller PF, Day JP, Bottiglier T, Braganza JM. Antioxidant therapy for recurrent pancreatitis: biochemical profiles in a placebocontrolled trial. Aliment Pharmacol Ther. 1992;6:229-40.

24. Saito I, Asano T, Sano K, Takakura K, Abe H, Yoshimoto T, et al. Neuroprotective effect of an antioxidant, ebselen, in patients with delayed neurological deficits after aneurysmal subarachnoid hemorrhage. Neurosurgery. 1998;42:269-77.

25. Yamaguchi T, Sano K, Takakura K, Saito I, Shinohara Y, Asano T, Ebselen Study Group, et al. Ebselen in acute ischemic stroke: a placebo-controlled, double-blind clinical trial. Stroke. 1998;29:12-7.

26. Heaney AP, Sharer N, Rameh B, Braganza JM, Durrington PN. Prevention of recurrent pancreatitis in familial lipoprotein lipase deficiency with high-dose antioxidant therapy. J Clin Endocrinol Metab. 1999:84:1203-5.
27. Ogawa A, Yoshimoto T, Kikuchi H, Sano K, Saito I, Yamaguchi T, et al. Ebselen in acute middle cerebral artery occlusion: a placebo-controlled, double-blind clinical trial. Cerebrovasc Dis. 1999;9:112-8.

28. Siriwardena AK, Mason JM, Balachandra S, Bagul A, Galloway S, Formela L, et al. Randomised, double blind, placebo controlled trial of intravenous antioxidant (n-acetylcysteine, selenium, vitamin C) therapy in severe acute pancreatitis. Gut. 2007:56:1439-44.

29. van Stijn MF, Ligthart-Melis GC, Boelens PG, Scheffer PG, Teerlink T, Twisk JW, et al. Antioxidant enriched enteral nutrition and oxidative stress after major gastrointestinal tract surgery. World J Gastroenterol. 2008;14:6960-9.

30. Lehmann C, Egerer K, Weber M, Krausch D, Wauer H, Newie T, et al. Effect of selenium administration on various laboratory parameters of patients at risk for sepsis syndrome [in German]. Med Klin (Munich). 1997;15 Suppl 3: 14-6.

31. Manzanares W, Biestro A, Galusso F, Torre MH, Mañáy N, Facchin G, et al. High-dose selenium for critically ill patients with systemic inflammation: pharmacokinetics and pharmacodynamics of selenious acid: a pilot study. Nutrition. 2010;26:634-40.

32. Kuklinski B, Buchner M, Müller T, Schweder R. Anti-oxidative therapy of pancreatitis - an 18-month interim evaluation [in German]. Z Gesamte Inn Med. 1992:47:239-45.

33. Angstwurm MW, Schopohl J, Gaertner R. Selenium substitution has no direct effect on thyroid hormone metabolism in critically ill patients. Eur J Endocrinol. 2004;151:47-54

34. Berger MM, Binnert C, Chiolero RL, Taylor W, Raffoul W, Cayeux MC, et al. Trace element supplementation after major burns increases burned skin trace element concentrations and modulates local protein metabolism but not whole-body substrate metabolism. Am J Clin Nutr. 2007;85:1301-6.

35. Sawyer MA, Mike JJ, Chavin K, Marino PL. Antioxidant therapy and survival in ARDS [abstract]. Crit Care Med. 1989;17 Suppl:S153.

36. Janka V, Ladislav K, Jozef F, Ladislav V. Restoration of antioxidant enzymes in the therapeutic use of selenium in septic patients. Wien Klin Wochenschr. 2013;125:316-25.

37. Schneider A, Markowski A, Momma M, Seipt C, Luettig B, Hadem J, et al. Tolerability and efficacy of a low-volume enteral supplement containing key nutrients in the critically ill. Clin Nutr. 2011;30:599-603.

38. Kuklinski B, Buchner M, Schweder R, Nagel R. Acute pancreatitis - a free radical disease: decrease in fatality with sodium selenite $\left(\mathrm{Na}_{2} \mathrm{SeO}_{3}\right)$ therapy [in German]. Z Gesamte Inn Med. 1991;46:145-9.

39. Zimmermann $T$, Albrecht $\mathrm{S}$, Kühne $H$, Vogelsang U, Grützmann R, Kopprasch S. Selenium administration in patients with sepsis syndrome: a prospective randomized study [in German]. Med Klin (Munich). 1997;92 Suppl 3:3-4.

40. Berger MM, Spertini F, Shenkin A, Wardle C, Wiesner L, Schindler C, et al. Trace element supplementation modulates pulmonary infection rates after major burns: a double-blind, placebo-controlled trial. Am J Clin Nutr. 1998; 68:365-71.

41. Angstwurm MW, Schottdorf J, Schopohl J, Gaertner R. Selenium replacement in patients with severe systemic inflammatory response syndrome improves clinical outcome. Crit Care Med. 1999:27:1807-13.

42. Porter JM, Ivatury RR, Azimuddin K, Swami R. Antioxidant therapy in the prevention of organ dysfunction syndrome and infectious complications after trauma: early results of a prospective randomized study. Am Surg. 1999:65:478-83.

43. Berger MM, Recmond MJ, Shenkin A, Rey F, Wardle C, Cayeux C, et al. Influence of selenium supplements on the post-traumatic alterations of the thyroid axis: a placebo-controlled trial. Intensive Care Med. 2001;27:91-100.

44. Lindner D, Lindner J, Baumann G, Dawczynski H, Bauch K. Investigation of antioxidant therapy with sodium selenite in acute pancreatitis: a prospective randomized blind trial [in German]. Med Klin (Munich). 2004;99: 708-12.

45. Angstwurm MW, Engelmann L, Zimmermann T, Lehmann C, Spes $\mathrm{CH}$, Abel $P$, et al. Selenium in Intensive Care (SIC): results of a prospective randomized, placebo-controlled, multiple-center study in patients with severe systemic inflammatory response syndrome, sepsis, and septic shock. Crit Care Med. 2007;35:118-26.

46. Berger MM, Baines M, Raffoul W, Benathan M, Chiolero RL, Reeves C, et al. Trace element supplementation after major burns modulates antioxidant status and clinical course by way of increased tissue trace element concentrations. Am J Clin Nutr. 2007;85:1293-300.

47. Forceville X, Laviolle B, Annane D, Vitoux D, Bleichner G, Korach JM, et al. Effects of high doses of selenium, as sodium selenite, in septic shock: a 
placebo-controlled, randomized, double-blind, phase II study. Crit Care. 2007;11:R73.

48. Mishra V, Baines M, Perry SE, McLaughlin PJ, Carson J, Wenstone R, et al. Effect of selenium supplementation on biochemical markers and outcome in critically ill patients. Clin Nutr. 2007;26:41-50.

49. Berger MM, Soguel L, Shenkin A, Revelly JP, Pinget C, Baines M, et al. Influence of early antioxidant supplements on clinical evolution and organ function in critically ill cardiac surgery, major trauma, and subarachnoid hemorrhage patients. Crit Care. 2008;12:R101.

50. El-Attar M, Said M, El-Assal G, Sabry NA, Omar E, Ashour L. Serum trace element levels in COPD patient: the relation between trace element supplementation and period of mechanical ventilation in a randomized controlled trial. Respirology. 2009;14:1180-7.

51. Montoya González C, Hernández Luna A, Villalobos Silva JA, Olvera Guzmán C, Aguirre Sánchez J, Granillo JF. Efecto antiinflamatorio del selenio en pacientes sépticos. Revista de la Asociación Mexicana de Medicina Crítica y Terapia Intensiva. 2009;23:199-205.

52. Andrews PJ, Avenell A, Noble DW, Campbell MK, Croal BL, Simpson WG, Scottish Intensive care Glutamine or seleNium Evaluative Trial Trials Group, et al. Randomised trial of glutamine, selenium, or both, to supplement parenteral nutrition for critically ill patients. BMJ. 2011;342:d1542.

53. Manzanares W, Biestro A, Torre MH, Galusso F, Facchin G, Hardy G. Highdose selenium reduces ventilator-associated pneumonia and illness severity in critically ill patients with systemic inflammation. Intensive Care Med. 2011;37:1120-7.

54. Valenta J, Brodska H, Drabek T, Hendl J, Kazda A. High-dose selenium substitution in sepsis: a prospective randomized clinical trial. Intensive Care Med. 2011;37:808-15.

55. Vincent $J \mathrm{~L}$, Forceville $X$. Critically elucidating the role of selenium. Curr Opin Anaesthesiol. 2008;21:148-54.

56. Jeong DW, Yoo MH, Kim TS, Kim IY. Protection of mice from allergen induced asthma by selenite: prevention of eosinophil infiltration by inhibition of NF-KB activation. J Biol Chem. 2002;277:17871-6.

57. Maehira F, Miyagi I, Eguchi Y. Selenium regulates transcription factor NF-KB activation during the acute phase reaction. Clin Chem Acta. 2003;334:163-71.

58. Chung YW, Kim TS, Lee SY, Lee SH, Choi Y, Kim N, et al. Selenite-induced apoptosis of osteoclasts mediated by the mitochondrial pathway. Toxicol Lett. 2006;160:143-50.

59. Wang Z, Forceville $X$, Van Antwerpen P, Piagnerelli M, Ahishakiye D, Macours $P$, et al. A large bolus, but not a continuous infusion, of sodium selenite improves outcome in peritonitis. Shock. 2009;32:140-6.

60. Berger MM. Micronutrients: myths and realities [in French]. Rev Med Suisse. 2011;7:230.

61. Fessler TA. Trace elements in parenteral nutrition: a practical guide for dosage and monitoring for adult patients. Nutr Clin Pract. 2013;28:722-31.

62. Stoppe C, Schälte G, Rossaint R, Coburn M, Graf B, Spillner J, et al. The intraoperative decrease of selenium is associated with the postoperative development of multiorgan dysfunction in cardiac surgical patients. Crit Care Med. 2011;39:1879-85.

63. Stoppe C, Spillner J, Rossaint R, Coburn M, Schälte G, Wildenhues A, et al. Selenium blood concentrations in patients undergoing elective cardiac surgery and receiving perioperative sodium selenite. Nutrition. 2013;29:158-65.

64. Stoppe C, McDonald B, Rex S, Manzanares W, Whitlock R, Fremes S, et al. SodiUm SeleniTe Administration IN Cardiac Surgery (SUSTAIN CSX-trial): study design of an international multicenter randomized double-blinded controlled trial of high dose sodium-selenite administration in high-risk cardiac surgical patients. Trials. 2014;15:339.

65. Critical Care Nutrition at Clinical Evaluation Research Unit, Kingston General Hospital/Queen's University. http://www.criticalcarenutrition.com/cpgs. Accessed 16 Apr 2016

\section{Submit your next manuscript to BioMed Central and we will help you at every step:}

- We accept pre-submission inquiries

- Our selector tool helps you to find the most relevant journal

- We provide round the clock customer support

- Convenient online submission

- Thorough peer review

- Inclusion in PubMed and all major indexing services

- Maximum visibility for your research

Submit your manuscript at www.biomedcentral.com/submit
) Biomed Central 